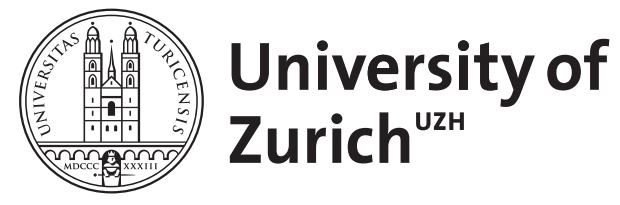

Zurich Open Repository and Archive

University of Zurich

University Library

Strickhofstrasse 39

CH-8057 Zurich

www.zora.uzh.ch

Year: 2008

\title{
Special issue on quantitative neuron modeling
}

Jolivet, Renaud ; Roth, Arnd ; Schürmann, Felix ; Gerstner, Wulfram ; Senn, Walter

DOI: https://doi.org/10.1007/s00422-008-0274-5

Posted at the Zurich Open Repository and Archive, University of Zurich

ZORA URL: https://doi.org/10.5167/uzh-156189

Journal Article

Published Version

Originally published at:

Jolivet, Renaud; Roth, Arnd; Schürmann, Felix; Gerstner, Wulfram; Senn, Walter (2008). Special issue on quantitative neuron modeling. Biological Cybernetics, 99(4-5):237-239.

DOI: https://doi.org/10.1007/s00422-008-0274-5 


\title{
Special issue on quantitative neuron modeling
}

\author{
Renaud Jolivet • Arnd Roth · Felix Schürmann • \\ Wulfram Gerstner • Walter Senn
}

Published online: 5 November 2008

(c) Springer-Verlag 2008

Single neurons are the fundamental constituents of all nervous systems. Understanding the properties and dynamics of single neurons is therefore one of the key challenges in modern neuroscience. Since the work of Lapicque (1907) [see also the recent translation by Brunel and van Rossum (2007)], single neuron models have enjoyed great popularity and have been the subject of many theoretical studies. Two broad categories of spiking neuron models have been extensively studied and used: Hodgkin-Huxley-type neuron models and simplified phenomenological neuron models, of which the integrate-and-fire model is the most famous representative. Each model type presents its own set of advantages and drawbacks. The Hodgkin-Huxley framework permits the design of detailed, biophysically realistic models, which can be used to study the effects of different types of ion channels including their distribution along dendrites. However, these models are computationally expensive and

R. Jolivet $(\varangle)$

Institute of Pharmacology and Toxicology,

University of Zurich, Zurich, Switzerland

e-mail: rjolivet@pharma.uzh.ch

\author{
A. Roth \\ Wolfson Institute for Biomedical Research, \\ University College London, Gower Street, \\ London WC1E 6BT, UK \\ e-mail: arnd.roth@ucl.ac.uk \\ F. Schürmann · W. Gerstner \\ Brain Mind Institute, EPFL, Lausanne, Switzerland \\ e-mail: felix.schuermann@epfl.ch \\ W. Gerstner \\ e-mail: wulfram.gerstner@epfl.ch \\ W. Senn \\ Department of Physiology, University of Bern, \\ Bern, Switzerland \\ e-mail: senn@pyl.unibe.ch
}

their analysis is usually difficult. Simple phenomenological neuron models, on the other hand, are analytically tractable and computationally cheap, but their realism is questionable. Both types of models have been used in countless studies of single neurons and network models, with the ultimate aim of understanding how information processing in single neurons and neural circuits gives rise to behaviour.

Despite the tremendous popularity both types of models enjoy, the question of their quantitative accuracy in reproducing experimentally measured neuronal dynamics has been barely addressed until recently. It was implicitly assumed that Hodgkin-Huxley-type neuron models are the most realistic, while simplified phenomenological neuron models were overlooked in that regard but extensively used because of their simplicity. However, are either of these models able to predict the rate and timing of output spikes of a real neuron, given arbitrary patterns of synaptic input? Can they correctly predict the result of the interactions between dendrites and soma? And can they quantitatively predict the effect of various intrinsic neuronal mechanisms, for example those underlying adaptation or bursting? With recent initiatives such as the Blue Brain Project (Markram 2006) or the recent large-scale model by Izhikevich and Edelman (2008), it is now time to try to answer these questions. In order to provide the community with a benchmark to address these questions, we set up an international scholarly challenge on quantitative single neuron modeling ${ }^{1}$. The present Special Issue comprises a selection of papers that address precisely these questions, and extensively describe the challenge: can Hodgkin-Huxley-type neuron models or simplified phenomenological neuron models predict the activity of real neurons? What is the minimal level of description required to achieve a reasonable accuracy of predictions made by

\footnotetext{
${ }^{1}$ http://lcn.epfl.ch/QuantNeuronMod/.
} 
the different models? What are the best strategies, algorithms, mapping techniques to automatically estimate model parameters based on experimental measurements? And finally, how reliable are the biological neurons themselves?

With the advent of powerful computers and software, tuning of single neuron models "by hand" and comparison of model output with experimental data "by eye" should be a thing of the past. The paper by Van Geit et al. (2008) gives an overview of current techniques for automated optimization of neuron models and describes different error functions that have been used to automatically compare and evaluate model responses with respect to experimental data. It also describes NeuroFitter, an open software package written for this purpose. The importance of using the correct error function to evaluate and optimize single neuron models is highlighted in the paper by Druckmann et al. (2008). They show that error functions which successfully lead an optimization algorithm to the (known) global optimum when fitting "synthetic" data generated by the same class of models can fail badly when they are applied to real experimental data. Finally, the paper by Pospischil et al. (2008) describes a practical application of these methods to data from four different electrophysiological classes of neurons.

The paper by Badel et al. (2008) describes a recently introduced method for the efficient generation of reduced neuron models from experimental data. Badel et al. elegantly demonstrate that the dynamic I-V curve of a neuron can be used to automatically fit a non-linear integrate-and-fire model. An attractive feature of their method is that it can be used in spike-triggered mode to quantify the distinct patterns of post-spike refractoriness seen in different classes of cortical neurons. On a similar line, the paper by De Lange et al. (2008) discusses fitting strategies and performances of the Hindmarsh-Rose model. De Lange et al. show that the Hindmarsh-Rose model can predict the spiking response of rat layer five neocortical pyramidal neurons with a precision comparable to the best known spiking models. The paper by Naud et al. (2008) gives a detailed account of the properties of the recently introduced adaptive exponential integrate-andfire neuron model. Naud etal. show that this simple neuron model can generate multiple firing patterns depending on the choice of parameter values and they present a phase diagram describing the transition from one firing type to another. They also provide an analytical criterion to distinguish between continuous adaption, initial bursting, regular bursting and two types of tonic spiking. Interestingly, this model is capable of producing irregular spiking when stimulated with constant current, indicating low-dimensional chaos. The adaptive exponential integrate-and-fire model is also the main topic of the paper by Touboul and Brette (2008). In their paper, the subthreshold features of the model are related to the dynamical properties of the differential system and the spike patterns to the properties of a Poincaré map defined by the sequence of spikes. The bifurcation structure is discussed. Lansky and Ditlevsen (2008) review methods for the estimation of input signals in stochastic diffusion leaky integrate-and-fire neuronal models. Strategies and performance of these methods are discussed for both the OrnsteinUhlenbeck model and the Feller model and for both intracellular and extracellular recordings.

Applications of simplified phenomenological neuron models are discussed in the last group of papers in the present Special Issue. The paper by Gollisch and Meister (2008) discusses the application of phenomenological "linear-nonlinear" models to the early visual system. In particular, it reviews new developments in modeling neuronal responses in the early visual system when the input to a particular ganglion cell is composed of combined ON-type as well as OFF-type bipolar cells. This type of input has been shown to lead to intriguing response characteristics that cannot be captured by a single linear filter. The paper by Richardson (2008) presents a novel numerical scheme to calculate exact results for the first-passage-time density and spike-train spectrum of the general class of non-linear integrate-and-fire models. The linear response properties and emergent states of recurrent networks are also derived. In companion papers, $\mathrm{La}$ Camera etal. (2008) and Giugliano et al. (2008) discuss the application of a mean field approach to the study of the collective dynamics of interacting neurons. More specifically, the paper by La Camera reviews theoretical and experimental results on quasi-stationary neural responses to noisy inputs with stationary statistics. The authors conclude that modified integrate-and-fire neuron models are good enough to reproduce faithfully all the relevant dynamical aspects of the neuronal response measured in experiments on real neurons in vitro. The applications to time-varying spike trains are reviewed in the companion paper by Giugliano et al. The roles of temporal and spatial input modulations in shaping output spike trains under in vivo-like input currents are discussed. To that end, the dynamic mean field theory of firing rate response is used and several experimental features are discussed.

It is conceivable that some features of the input to a neuron are more important than others, and that the input current contains a mixture of signal and noise. How can we assess the performance of a model in such a case? The approach presented by London et al. (2008) is to define a certain input as the signal and measure its efficacy in influencing spike output using information theoretical quantities. The mutual information between the spike train measured in the real neuron and the spike train predicted by the model is introduced as an alternative criterion to evaluate model performance. Information theory is also employed in the paper by Koepsell and Sommer (2008). They present a model describing the periodicity observed in measured neural spike trains, and 
an information theoretical approach to measure information in oscillatory activity.

Finally, the paper by Jolivet etal. (2008) describes the recently established quantitative single-neuron modeling competition and presents the results received over the first 2 years (2007-2008). Based on these results, the paper discusses two fundamental questions for future developments in the field, namely what is a good evaluation criterion and what is a good neuron model.

The contributions to this Special Issue are representative of the state-of-the-art in the field and indicate new directions that will be followed in the years to come. As more and more neuroscientists move to a more quantitative description of neuronal activity, we expect this Special Issue to provide students and young researchers with reference readings in this rapidly growing area of research.

\section{References}

Badel L, Lefort S, Petersen CCH, Gerstner W, Richardson MJE (2008) Extracting nonlinear integrate-and-fire models from experimental data using dynamic I-V curves. Biol Cybern 99. doi:10.1007/ s00422-008-0259-4

Brunel N, van Rossum MCW (2007) Lapicque's 1907 paper: from frogs to integrate-and-fire. Biol Cybern 97(5):337-339

De Lange E, Hasler M (2008) Predicting single spikes and spike patterns with the Hindmarsh-Rose model. Biol Cybern 99. doi:10.1007/ s00422-008-0260-y

Druckmann S, Berger TK, Hill S, Schürmann F, Markram H, Segev I (2008) Evaluating automated parameter constraining procedures of neuron models by experimental and surrogate data. Biol Cybern 99. doi:10.1007/s00422-008-0269-2

Giugliano M, La Camera G, Fusi S, Senn W (2008) The response of cortical neurons to in vivo-like input current: temporal and spatial input modulations. II. Time-varying responses network dynamics somato-dendritic interactions. Biol Cybern 99. doi:10.1007/ s00422-008-0270-9
Gollisch T, Meister M (2008) Modeling convergent ON and OFF pathways in the early visual system. Biol Cybern 99. doi:10.1007/ s00422-008-0252-y

Izhikevich EM, Edelman GM (2008) Large-scale model of mammalian thalamocortical systems. Proc Natl Acad Sci USA 105(9):35933598

Jolivet R, Schürmann F, Berger TK, Naud R, Gerstner W, Roth A (2008) The quantitative single-neuron modeling competition. Biol Cybern 99. doi:10.1007/s00422-008-0261-x

Koepsell K, Sommer FT (2008) Information transmission in oscillatory neural activity. Biol Cybern 99. doi:10.1007/s00422-008-0273-6

La Camera G, Giugliano M, Senn W, Fusi S (2008) The response of cortical neurons to in vivo-like input current: theory and experiment. I. quasi-stationary spike trains. Biol Cybern 99. doi:10. 1007/s00422-008-0272-7

Lansky P, Ditlevsen S (2008) A review of the methods for signal estimation in stochastic diffusion leaky integrate-and-fire neuronal models. Biol Cybern 99. doi:10.1007/s00422-008-0237-x

Lapicque L (1907) Recherches quantitatives sur l'excitation électrique des nerfs traitée comme une polarisation. J Physiol Pathol Gen 9:620-635

London M, Larkum ME, Häusser M (2008) Predicting the synaptic information efficacy in cortical layer 5 pyramidal cells using a minimal integrate-and-fire model. Biol Cybern 99. doi:10.1007/ s00422-008-0268-3

Markram H (2006) The blue brain project. Nat Rev Neurosci 7(2):153160

Naud R, Marcille N, Clopath C, Gerstner W (2008) Firing patterns in the adaptive exponential integrate-and-fire model. Biol Cybern 99. doi:10.1007/s00422-008-0264-7

Pospischil M, Toledo-Rodriguez M, Monier C, Piwkowska Z, Bal T, Frégnac Y, Markram H, Destexhe A (2008) Minimal HodgkinHuxley type models for different classes of cortical and thalamic neurons. Biol Cybern 99. doi:10.1007/s00422-008-0263-8

Richardson MJE (2008) Spike-train spectra and network response functions for non-linear integrate-and-fire neurons. Biol Cybern 99. doi:10.1007/s00422-008-0244-y

Touboul J, Brette R (2008) Dynamics and bifurcations of the adaptive exponential integrate-and-fire model. Biol Cybern 99. doi:10. 1007/s00422-008-0267-4

Van Geit W, De Schutter E, Achard P (2008) Automated neuron model optimization techniques: a review. Biol Cybern 99. doi:10.1007/ s00422-008-0257-6 